\title{
The complete mitogenome of Curculio chinensis (Chevrolat, 1878) (Coleoptera: Curculionidae: Curculioninae)
}

\author{
Kai Hü, Nian-nian Zhang ${ }^{\ddagger}$, Zai-Hua Yang ${ }^{\ddagger}$ \\ ‡ Guizhou Academy of Forestry, Guiyang, China
}

Corresponding author: Zai-Hua Yang (yangzaihua008@126.com)

Academic editor: Jennifer C. Girón Duque

Received: 27 May 2021 | Accepted: 08 Oct 2021 | Published: 25 Oct 2021

Citation: Hu K, Zhang N-n, Yang Z-H (2021) The complete mitogenome of Curculio chinensis (Chevrolat, 1878)

(Coleoptera: Curculionidae: Curculioninae). Biodiversity Data Journal 9: e69196.

https://doi.org/10.3897/BDJ.9.e69196

\begin{abstract}
The mitogenome of Curculio chinensis (Chevrolat, 1878) was sequenced and annotated to better identify $C$. chinensis and related species. The mitogenome is $18,680 \mathrm{bp}$ in length, includes the 37 typical mitochondrial genes (13 protein-coding genes, two ribosomal RNA genes and 22 transfer RNA genes) and two control regions (total length: 3,879 bp). Mitogenome organisation, nucleotide composition and codon usage are similar to the previously sequenced Curculio mitogenomes. All 13 protein-coding genes use ATN or TTG as start codon and end with TAA/G or incomplete stop codons (single T-). Twenty-one transfer RNA genes have the typical clover-leaf structure, while the dihydrouridine (DHU) arm of trnS1 is missing. In Curculio mitogenomes, the size of the control region is highly variable. Both $\mathrm{ML}$ and $\mathrm{BI}$ analyses, based on the 13 PCGs and two rRNAs from six species of Curculioninae, strongly supported the monophyly of Curculio. In Curculio, the relationships amongst included species were inferred as ((C. chinensis + Curculio. sp. $)+$ (Curculio davidi + Curculio elephas)), with C. chinensis and C. sp. forming a clade $(B S=100 ; P P=1)$.
\end{abstract}




\section{Keywords}

mitochondrial genome, camellia weevil, phylogenetic analysis, secondary structure

\section{Introduction}

The typical mitogenome of insects is a circular double-stranded DNA molecule with 15-18 $\mathrm{kb}$ in length, encoding 13 protein-coding genes (PCGs), two ribosomal RNA genes (rRNAs), 22 transfer RNA genes (tRNAs) and also includes a large non-coding region (control region) (Boore 1999, Cameron 2014). In insects, the mitogenome has been widely used as a molecular marker to explore population genetics, phylogeny and evolution (Fenn et al. 2008, Galtier et al. 2009, Cameron 2014).

The camellia weevil, Curculio chinensis (Chevrolat, 1878) is widely distributed in most of China's Camellia spp. (family Theaceae) producing areas (Li et al. 2015). It is one of the most serious pests of tea and causes huge economic losses (Li et al. 2015). Since different species exhibit distinct responses to specific biocontrol agents and pesticides, accurate species identification is very important in pest management (Zhou et al. 2020). However, the camellia weevil is often difficult to identify using morphological characteristics of the larvae. It is impractical to identify camellia weevil by rearing larvae to adults because the larvae are long-lived and difficult to rear when removed from the seed (Shu et al. 2013, He et al. 2014). Molecular identification has proven to be reliable and effective for specieslevel identification of insects at any life stage (Chen et al. 2016).

In this study, we sequenced and annotated the mitogenome of $C$. chinensis and analysed its characteristics. In addition, we reconstructed the molecular phylogenetic relationships of C. chinensis and other species of the genus Curculio. The molecular data presented here will be useful for studies on identification and evolution in $C$. chinensis and related species.

\section{Materials and methods}

\section{Sample collection and DNA extraction}

Adult specimens of $C$. chinensis were collected from Camellia spp. in Yunguanshan Forest Farm, Guiyang City, Guizhou Province, China $\left(26.48208727^{\circ} \mathrm{N}, 106.75480714^{\circ} \mathrm{E}\right.$, July 2020) (C. chinensis is a host-specifc predator of the seeds of Camellia spp.). All fresh specimens were preserved in $100 \%$ ethyl alcohol and deposited in a $-20^{\circ} \mathrm{C}$ freezer at the laboratory of Guizhou Academy of Forestry, Guiyang. Identification of adult specimens was based on morphological characteristics (Chao and Chen 1980). Whole genomic DNA was extracted from thorax muscle tissues using the Biospin Insect Genomic DNA Extraction Kit (BioFlux) following the manufacturer's instructions. Voucher specimens are stored in the insect collection of Guizhou Academy of Forestry. 


\section{Mitogenome sequencing, assembly, annotation and bioinformatic analyses}

The complete mitogenome of $C$. chinensis was sequenced using NGS (next-generation sequencing) (Illumina HiSeq X10; Biomarker Technologies Corporation, Beijing, China). About $1.26 \mathrm{~Gb}$ clean data were assembled into a complete circular mitogenome by NOVOPlasty v.2.7.0 (Dierckxsens et al. 2016) using the COX1 sequence of Curculio davidi Fairmaire, 1878 (GenBank accession: NC 034293) (Xu et al. 2017) as an initial seed. The mitogenome was annotated using MITOZ v.1.04 (Meng et al. 2019) and checked manually in Geneious v.8.1.3 (Biomatters, Auckland, New Zealand). The tRNA secondary structures were manually drawn using Adobe Illustrator CC2017, based on the MITOS Web Server (Bernt et al. 2013) predictions. The mitogenome map was drawn with the programme Organellar Genome DRAW (OGDRAW) (Lohse et al. 2013). Bioinformatic analyses, including nucleotide composition, composition skew, codon usage of PCGs, relative synonymous codon usage (RSCU) and mitogenomic organisation tables were conducted using PhyloSuite v.1.2.2 (Zhang et al. 2019).

\section{Molecular phylogenetic analysis}

A total of six mitogenomes from two genera of Curculioninae were used for the phylogenetic analyses (Table 1). We used as much mitogenome data for the genus Curculio in NCBI as possible. Of these, four species belong to Curculio (the ingroup), while the remaining two species from the genus Anthonomus Germar, 1817 were chosen as outgroup. Nucleotide sequences (without stop codons) for the 13 PCGs were aligned using MAFFT v.7 (Katoh and Standley 2013) with the G-INS-i (accurate) strategy and codon alignment mode (Code table: Invertebrate mitochondrial genetic codon). The rRNAs genes (rrnL and rrnS) were aligned using MAFFT v.7 (Katoh and Standley 2013) with the Q-INS-I algorithm (which takes account of the secondary structure of rRNA genes). Ambiguously aligned areas were removed using Gblocks v.0.91b (Talavera and Castresana 2007), respectively. Gene alignments were concatenated using PhyloSuite v.1.2.2 (Zhang et al. 2019). Partitioning scheme and nucleotide substitution models for Maximum Likelihood $(\mathrm{ML})$ and Bayesian Inference (BI) phylogenetic analyses were selected with PartitionFinder2 (Lanfear et al. 2017) using the Bayesian Information Criterion (BIC) (Suppl. materials 1, 2). ML analyses were reconstructed by IQ-TREE v.1.6.3 (Nguyen et al. 2015) under the ultrafast bootstrap (UFB) approximation approach (Minh et al. 2013) with 5,000 replicates. $\mathrm{BI}$ analysis was performed using MrBayes v.3.2.7a (Ronquist et al. 2012) in the CIPRES Science Gateway (Miller et al. 2010) with four chains (one cold chain and three hot chains). Two independent runs of 2,000,000 generations were carried out with sampling every 1,000 generations. The first $25 \%$ of trees were discarded as burn-in. After the average standard deviation of split frequencies fell below 0.01 , stationarity was assumed. 
Table 1.

Mitogenomes of the six Curculioninae taxa used in this study.

\begin{tabular}{|l|l|l|l|}
\hline Subfamily & Species & Accession number & Reference \\
\hline Curculioninae & Anthonomus eugenii & $\underline{\mathrm{NC} 044711}$ & van de Vossenberg et al. 2019 \\
\hline & Anthonomus rubi & $\underline{\mathrm{NC} 044714}$ & van de Vossenberg et al. 2019 \\
\hline Curculio chinensis & $\underline{\mathrm{MZ417388}}$ & This study \\
\hline Curculio sp. & $\underline{\mathrm{MG} 728095}$ & Unpublished \\
\hline Curculio davidi & $\underline{\mathrm{NC} 034293}$ & Xu et al. 2017 \\
\hline Curculio elephas & $\underline{\mathrm{KX087269}}$ & Unpublished \\
\hline
\end{tabular}

\section{Results and discussion}

\section{Mitogenome organisation and nucleotide composition}

The mitogenome of $C$. chinensis is a double-stranded circular DNA molecule, containing 37 typical mitochondrial genes (13 PCGs, 22 tRNAs and two rRNAs) and two control regions (Table 2, Fig. 1), which are common in Curculioninae mitogenomes (Narakusumo et al. 2020). The newly-sequenced mitogenome (length: $18,680 \mathrm{bp}$ ) is medium-sized compared to other Curculio mitogenomes (ranging from 16,852 bp Curculio davidi, GenBank accession: NC 034293 to 19,216 bp Curculio sp., GenBank accession: MG728095) (Xu et al. 2017). Variation in the size of the control region is the main source of the length variation in Curculio mitogenomes (Fig. 2). The mitogenome of $C$. chinensis has the same gene order as other previously sequenced Curculio species (Xu et al. 2017). A total of 71 overlapping nucleotides were found in ten pairs of neighbouring genes, the longest overlap (23 bp) being identified between the trnL1 and rrnL. Furthermore, there are 151 intergenic nucleotides dispersed across 13 gene boundaries and the longest intergenic region (103 bp) is located between trnS2 and nad1.

Table 2.

Mitogenomic organisation of C. chinensis.

\begin{tabular}{|c|c|c|c|c|c|c|c|}
\hline \multirow[t]{2}{*}{ Gene name } & \multicolumn{2}{|c|}{ Location } & \multirow[t]{2}{*}{ Size (bp) } & \multirow{2}{*}{$\begin{array}{l}\text { Intergenic } \\
\text { nucleotides }\end{array}$} & \multicolumn{2}{|c|}{ Codon } & \multirow[t]{2}{*}{ Strand } \\
\hline & From & To & & & Start & Stop & \\
\hline $\operatorname{trnl}$ & 1 & 65 & 65 & & & & + \\
\hline CR2 & 66 & 1947 & 1882 & & & & + \\
\hline $\operatorname{trn} Q$ & 1948 & 2016 & 69 & & & & - \\
\hline $\operatorname{trnM}$ & 2018 & 2085 & 68 & 1 & & & + \\
\hline nad2 & 2089 & 3096 & 1008 & 3 & ATA & TAA & + \\
\hline $\operatorname{trnW}$ & 3111 & 3174 & 64 & 14 & & & + \\
\hline $\operatorname{trnC}$ & 3174 & 3239 & 66 & -1 & & & - \\
\hline
\end{tabular}




\begin{tabular}{|c|c|c|c|c|c|c|c|}
\hline \multirow[t]{2}{*}{ Gene name } & \multicolumn{2}{|c|}{ Location } & \multirow[t]{2}{*}{ Size (bp) } & \multirow{2}{*}{$\begin{array}{l}\text { Intergenic } \\
\text { nucleotides }\end{array}$} & \multicolumn{2}{|c|}{ Codon } & \multirow[t]{2}{*}{ Strand } \\
\hline & From & To & & & Start & Stop & \\
\hline $\operatorname{trn} Y$ & 3242 & 3305 & 64 & 2 & & & - \\
\hline $\operatorname{cox} 1$ & 3298 & 4842 & 1545 & -8 & ATT & TAA & + \\
\hline $\operatorname{trnL2}$ & 4838 & 4902 & 65 & -5 & & & + \\
\hline $\operatorname{cox} 2$ & 4903 & 5586 & 684 & & ATT & TAA & + \\
\hline trnK & 5588 & 5658 & 71 & 1 & & & + \\
\hline $\operatorname{trnD}$ & 5661 & 5725 & 65 & 2 & & & + \\
\hline atp8 & 5726 & 5884 & 159 & & ATT & TAA & + \\
\hline atp6 & 5881 & 6552 & 672 & -4 & ATA & TAA & + \\
\hline $\operatorname{cox} 3$ & 6563 & 7343 & 781 & 10 & ATT & $\mathrm{T}$ & + \\
\hline $\operatorname{trnG}$ & 7344 & 7407 & 64 & & & & + \\
\hline nad3 & 7408 & 7761 & 354 & & ATT & TAG & + \\
\hline $\operatorname{trn} A$ & 7760 & 7826 & 67 & -2 & & & + \\
\hline $\operatorname{trnR}$ & 7827 & 7888 & 62 & & & & + \\
\hline $\operatorname{trnN}$ & 7887 & 7950 & 64 & -2 & & & + \\
\hline $\operatorname{trnS} 1$ & 7951 & 8017 & 67 & & & & + \\
\hline $\operatorname{trnE}$ & 8025 & 8088 & 64 & 7 & & & + \\
\hline $\operatorname{trnF}$ & 8089 & 8153 & 65 & & & & - \\
\hline nad5 & 8137 & 9873 & 1737 & -17 & ATT & TAA & - \\
\hline $\operatorname{trnH}$ & 9874 & 9936 & 63 & & & & - \\
\hline nad4 & 9937 & 11272 & 1336 & & ATG & $\mathrm{T}$ & - \\
\hline nad4L & 11266 & 11559 & 294 & -7 & ATG & TAA & - \\
\hline $\operatorname{trnT}$ & 11562 & 11626 & 65 & 2 & & & + \\
\hline $\operatorname{trn} P$ & 11627 & 11692 & 66 & & & & - \\
\hline nad6 & 11695 & 12198 & 504 & 2 & ATT & TAA & + \\
\hline cob & 12202 & 13338 & 1137 & 3 & ATA & TAA & + \\
\hline $\operatorname{trnS2}$ & 13339 & 13405 & 67 & & & & + \\
\hline nad1 & 13509 & 14459 & 951 & 103 & TTG & TAG & - \\
\hline $\operatorname{trnL} 1$ & 14461 & 14525 & 65 & 1 & & & - \\
\hline $\mathrm{rrnL}$ & 14503 & 15831 & 1329 & -23 & & & - \\
\hline $\operatorname{trnV}$ & 15830 & 15895 & 66 & -2 & & & - \\
\hline rrns & 15896 & 16683 & 788 & & & & - \\
\hline CR1 & 16684 & 18680 & 1997 & & & & + \\
\hline
\end{tabular}

The nucleotide content of the Curculio mitogenomes exhibit strong AT bias: $76.9 \%-77.5 \%$ in the whole genome, $75.7 \%-76.1 \%$ in the PCGs, $76.8 \%-78.3 \%$ in the tRNAs, $76.9 \%-78.8 \%$ in the rRNAs and $78.8 \%-83.7 \%$ in the control region (Table 3 ). In every 
sequenced mitogenome of Curculio, PCGs have the lowest AT content, while the control region has the highest AT content (Table 3). All four Curculio mitogenomes have positive AT-skews (0.052-0.062) and negative GC-skews (-0.203 to -0.17$)$, similar to other recently reported weevil mitogenomes (Apriyanto and Tambunan 2020, Song et al. 2020, Wang et al. 2020, Wang et al. 2021) and most other insects (Wei et al. 2010).

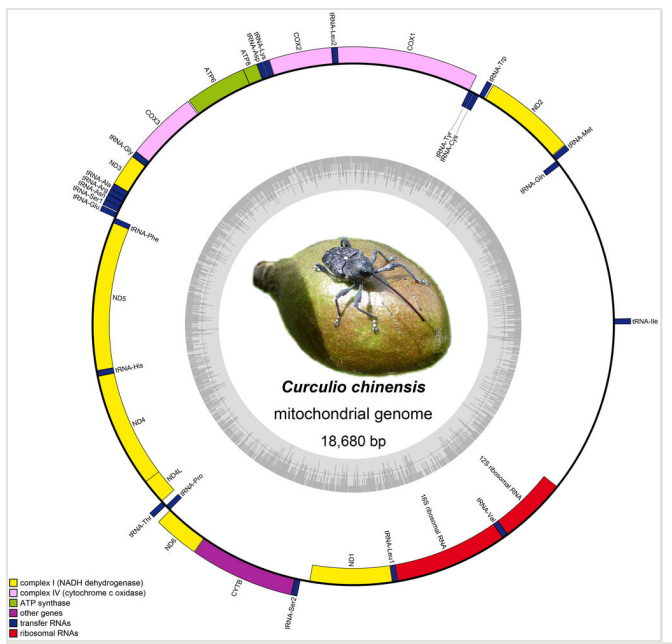

Figure 1. doi

Circular map of the mitogenome of $C$. chinensis. The outer circle shows the gene map of $C$. chinensis and the genes outside the map are coded on the major strand (J-strand), whereas the genes on the inside of the map are coded on the minor strand (N-strand). Genes are represented by different colour blocks.

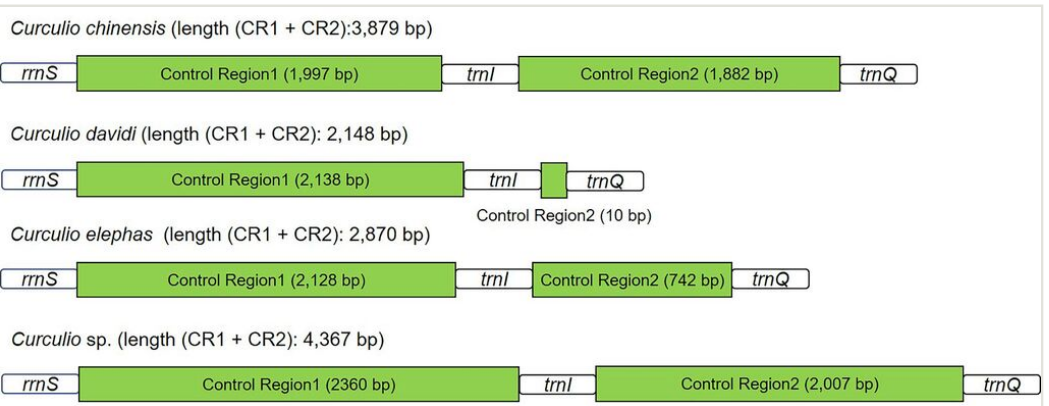

Figure 2. doi

Control regions in the four complete Curculio mitogenomes.

\section{Protein-coding genes}

The total size of all 13 PCGs of $C$. chinensis is $11,160 \mathrm{bp}$, accounting for $59.74 \%$ of the entire mitogenome (Table 3). In 13 PCGs, nad2, cox1, cox2, atp8, atp6, cox3, nad3, nad5, nad4, nad4L, nad6 and cob use ATN (ATA/T/G/C) as start codon, while nad1 is initiated by 
TTG, which is common for Curculio mitogenomes (Xu et al. 2017). All PCGs stopped with TAA/G or their incomplete form single T-. The incomplete termination codon single T- can be completed by post-transcriptional polyadenylation (Ojala et al. 1981). The AT-skews of all PCGs amongst Curculio range from -0.146 (C. davidi) (Xu et al. 2017) to -0.133 (C. chinensis and Curculio sp.), showing a biased use for the $T$ nucleotide. The relative synonymous codon usage (RSCU) of $C$. chinensis mitogenome is presented in Fig. 3, indicating Leu, Phe and Ile are the three most frequently used amino acids. In the new mitogenome, the four most frequently utilised codons are UUA-Leu, UUU-Phe, AUU-lle and AUA-Met. The most frequently used codons are composed of $A$ nucleotide or $U$ nucleotide, which reflects the high AT content of PCGs.

Table 3.

Base composition and skewness of mitogenomes of Curculio chinensis, Curculio sp., Curculio davidi and Curculio elephas.

\begin{tabular}{|l|l|l|l|l|}
\hline Feature & Length & A+T\% & AT-skew & GC-skew \\
\hline C. chinensis, C. sp., C. davidi and C. elephas & & \\
\hline $\begin{array}{l}\text { Whole } \\
\text { genome }\end{array}$ & $18680 / 19216 / 16852 / 17591$ & $76.9 / 77 / 77.2 / 77.5$ & $0.056 / 0.06 / 0.062 / 0.052$ & $-0.19 /-0.203 /-0.185 /-0.17$ \\
\hline PCGs & $11160 / 11091 / 11154 / 10989$ & $76 / 75.7 / 75.9 / 76.1$ & $-0.133 /-0.133 /-0.146 /-0.145$ & $-0.038 /-0.043 /-0.049 /-0.055$ \\
\hline tRNAs & $1442 / 1444 / 1440 / 1447$ & $76.8 / 77.8 / 76.8 / 78.3$ & $0.036 / 0.02 / 0.011 / 0.026$ & $0.12 / 0.121 / 0.12 / 0.144$ \\
\hline rRNAs & $2117 / 2059 / 2152 / 2084$ & $78.8 / 78.3 / 76.9 / 78.3$ & $-0.064 /-0.086 /-0.062 /-0.063$ & $0.345 / 0.348 / 0.315 / 0.307$ \\
\hline CR1 & $1997 / 2360 / 2138 / 2128$ & $84.2 / 83 / 83.7 / 85.6$ & $-0.014 / 0.058 / 0.089 / 0.058$ & $-0.478 /-0.606 /-0.24 /-0.24$ \\
\hline CR2 & $1882 / 2007 / 10 / 742$ & $71.4 / 73.9 / 90 / 69$ & $-0.023 /-0.076 /-0.333 /-0.011$ & $0.235 / 0.179 /-1 / 0.139$ \\
\hline $\begin{array}{l}\text { Control } \\
\text { region } \\
\text { (CR1 + }\end{array}$ & $3879 / 4367 / 2148 / 2870$ & $78 / 78.8 / 83.7 / 81.3$ & $-0.018 / 0 / 0.087 / 0.043$ & $-0.028 /-0.161 / 0.234 /-0.078$ \\
\hline CR2) & & & & \\
\hline
\end{tabular}

\section{Transfer and ribosomal RNA genes}

The typical sets of 22 tRNAs were identified with the size ranging from $62 \mathrm{bp}$ (trnR) to 71 bp (trnK) (Table 2). The AT content of tRNAs (76.8\%-78.3\%) was slightly higher than that of the PCGs (75.7\%-76.1\%) (Table 3). Most tRNAs have clover-leaf secondary structures, except for trnS1, where the dihydrouridine (DHU) arm became a simple loop (Fig. 4). This feature is common in metazoan mitogenomes (Garey and Wolstenholme 1989). A total of 30 mismatched base pairs belonging to six types ( $U-G, U-U, A-C, A-G, U-C$ and $A-A$ ) were found in the arm structures of the 22 tRNAs.

The length of rrnS and rrnL genes ranges from 2,059 bp (C. sp.) to 2,152 bp (C. chinensis) and AT content of rRNAs is conserved in the Curculio (Table 3). For $C$. chinensis, the rrnL gene (length: $1329 \mathrm{bp}$ ) is encoded between trnL1 and trnV and the rrnS gene (length: 788 $\mathrm{bp}$ ) is encoded between trnV and the control region, similar to other sequenced Curculio (Xu et al. 2017). 


\section{Control region}

The control region regulates the replication and transcription of mtDNA (Boore 1999, Cameron 2014). In each sequenced Curculio mitogenome, the control region is subdivided by trnl into two parts (control region1 and control region2) (Fig. 2). Control region1 (CR1) is located between rrnS and trnl, while control region2 (CR2) is located between trnl and trnQ. The length and AT content of CR1 (1,997-2,360 bp and 83\%-85.6\%) are slightly higher than CR2 (10-2,007 bp and 60\%-90\%) (Table 3).

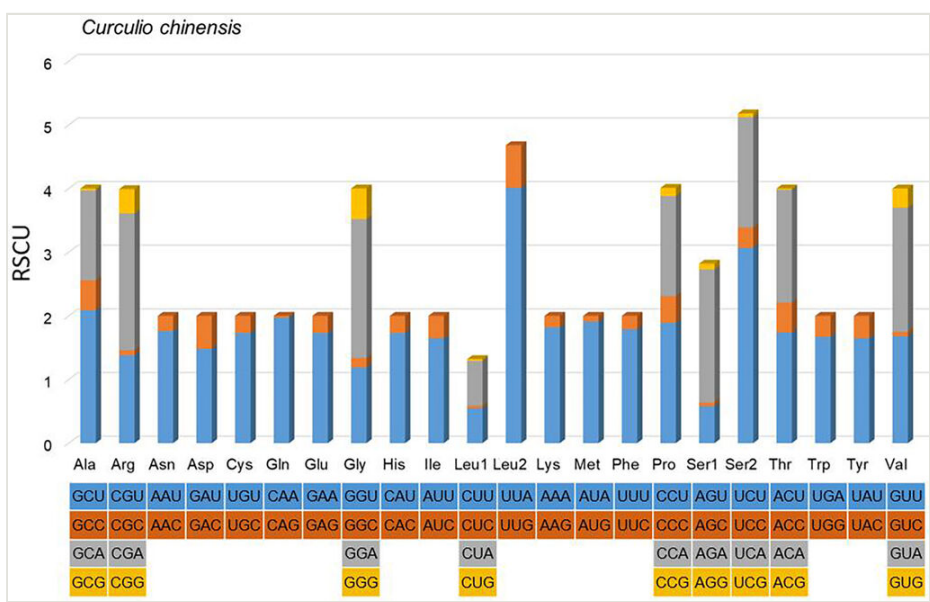

Figure 3. doi

Relative synonymous codon usage (RSCU) of the mitogenome of C. chinensis. The stop codon is not shown.

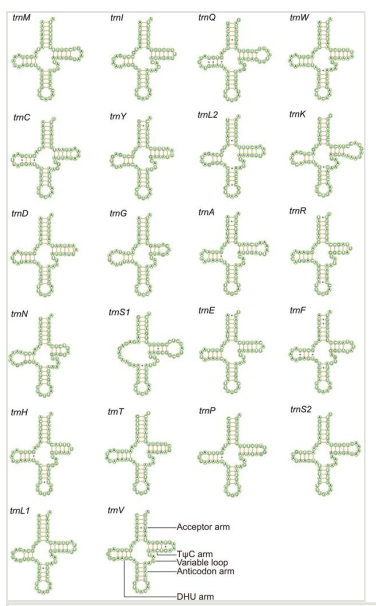

Figure 4. doi

Secondary structures of 22 tRNAs in the mitogenome of C. chinensis. Lines (-) indicate Watson-Crick base pairings, whereas dots $(\cdot)$ indicate unmatched base pairings. 


\section{Phylogenetic relationships}

Based on $\mathrm{ML}$ and $\mathrm{BI}$ analyses of nucleotide data of 13 PCGs and two rRNAs, we reconstructed the phylogenetic relationships of four species of Curculio. The trees of both analyses have congruent topologies, with all branches strongly supported (Fig. 5). Furthermore, relationships recovered in our analyses are similar to those found by Song et al. (Song et al. 2020), but we only focused on the phylogenetic relationships within Curculio. The monophyly of the genus Curculio was recovered with strong support, consistent with the previous study (Song et al. 2020). In Curculio, the relationships amongst included species were inferred as $((C$. chinensis + C. sp. $)+($ Curculio davidi + Curculio elephas Fabricius, 1781)), with C. chinensis and C. sp. forming a clade. In China's Camellia spp. producing areas, both $C$. chinensis and C. sp. are host-specific predators of the seeds of Camellia spp. The topologies of the phylogenetic trees reconstructed by us strongly supported the sister relationship between these two Curculio species ( $B S=100$; $\mathrm{PP}=1$ ), which may reflect a convergent evolutionary phenomenon in Curculio species with Camellia spp. as their host.

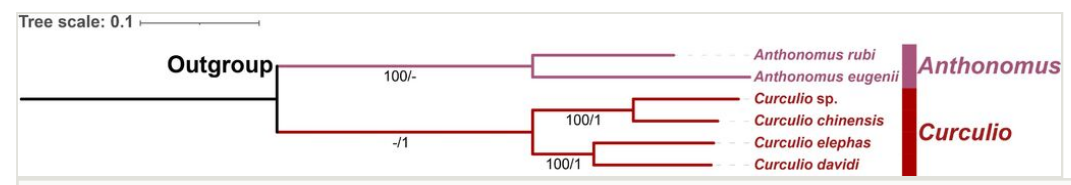

Figure 5. doi

$\mathrm{ML}$ and $\mathrm{BI}$ phylogenetic trees for Curculio, based on the nucleotide sequence data of 13 PCGs and two rRNAs from $C$. chinensis and other five species belonging to two related genera of Curculioninae. Bootstrap support values (BS) and Bayesian posterior probabilities (PP) are indicated on the branch.

\section{Acknowledgements}

This work was supported by the Service Enterprise Action Plan of Guizhou Provincial Camellia oleifera Team (QKHSEAP [2018] 4003), the Engineering Technology Research Center of Camellia oleifera of Guizhou Province (QKHPT [2018] 5252), the Effects of Plant Growth Regulators on the Flower and Fruit Protection of Camellia oleifera (QLKH [2019] 04) and the Project of Guizhou Science and Technology Platform and Talent Team Under Grant (nos. QKHPTRC [2018] 5610, QKHPTRC [2016] 5669).

\section{Author contributions}

The study was conceptualised by Zai-Hua Yang and Nian-nian Zhang organised the sample collection. Kai Hu conducted all the laboratory work. Kai Hu and Zai-Hua Yang have written the manuscript. 


\section{Conflicts of interest}

The authors report no conflicts of interest and are responsible for the content and writing of the paper.

\section{References}

- $\quad$ Apriyanto V, Tambunan VB (2020) The complete mitochondrial genome of oil palm pollinating weevil, Elaeidobius kamerunicus Faust. (Coleoptera: Curculionidae). Mitochondrial DNA Part B 5 (3): 3450-3452. https://doi.org/10.1080/238 $\underline{02359.2020 .1823899}$

- $\quad$ Bernt M, Donath A, Jühling F, Externbrink F, Florentz C, Fritzsch G, Pütz J, Middendorf M, Stadler PF (2013) MITOS: improved de novo metazoan mitochondrial genome annotation. Molecular Phylogenetics and Evolution 69 (2): 313-319. https://doi.org/ 10.1016/j.ympev.2012.08.023

- $\quad$ Boore JL (1999) Animal mitochondrial genomes. Nucleic Acids Research 27 (8): 1767-1780. https://doi.org/10.1093/nar/27.8.1767

- $\quad$ Cameron SL (2014) Insect mitochondrial genomics: implications for evolution and phylogeny. Annual Review of Entomology 59: 95-117. https://doi.org/10.1146/annurevento-011613-162007

- Chao YC, Chen YQ (1980) Economic insect fauna of China fasc. 20 Coleoptera: Curculionidae (1), 1st ed. Science Press, Beijing, 1-184 pp.

- $\quad$ Chen P, Zheng B, Liu J, Wei S (2016) Next-Generation Sequencing of Two Mitochondrial Genomes from Family Pompilidae (Hymenoptera: Vespoidea) Reveal Novel Patterns of Gene Arrangement. International Journal of Molecular Sciences 17 (10): 1641. https://doi.org/10.3390/ijms17101641

- Dierckxsens N, Mardulyn P, Smits G (2016) NOVOPlasty: de novo assembly of organelle genomes from whole genome data. Nucleic Acids Research 45 (4): e18. https://doi.org/10.1093/nar/gkw955

- $\quad$ Fenn JD, Song H, Cameron SL, Whiting MF (2008) A preliminary mitochondrial genome phylogeny of Orthoptera (Insecta) and approaches to maximizing phylogenetic signal found within mitochondrial genome data. Molecular Phylogenetics and Evolution 49 (1): 59-68. https://doi.org/10.1016/j.ympev.2008.07.004

- Galtier N, Nabholz B, Glemin S, Hurst GD (2009) Mitochondrial DNA as a marker of molecular diversity: a reappraisal. Molecular Ecology 18: 4541-4550. https://doi.org/ 10.1111/j.1365-294X.2009.04380.x

- $\quad$ Garey JR, Wolstenholme DR (1989) Platyhelminth mitochondrial DNA: evidence for early evolutionary origin of a tRNA ${ }^{\text {ser }}$ AGN that contains a dihydrouridine arm replacement loop, and of serine-specifying AGA and AGG codons. Journal of Molecular Evolution 28: 374-387. https://doi.org/10.1007/BF02603072

- He LH, Li ZW, Liu JJ, Si JY, Zeng AP (2014) Correlation between Damage of Curculio chinensis and Fruit Traits of Camellia meiocarpa. Scientia Silvae Sinicae 50 (12): 151-155. https://doi.org/10.11707/j.1001-7488.20141221

- $\quad$ Katoh K, Standley DM (2013) MAFFT multiple sequence alignment software version 7: improvements in performance and usability. Molecular Biology and Evolution 30 (4): 772-780. https://doi.org/10.1093/molbev/mst010 
- $\quad$ Lanfear R, Frandsen PB, Wright AM, Senfeld T, Calcott B (2017) PartitionFinder 2: new methods for selecting partitioned models of evolution for molecular and morphological phylogenetic analyses. Molecular Biology and Evolution 34 (3): 772-773. https://doi.org/ 10.1093/molbev/msw260

- $\quad$ Li ZW, He LH, Xia J, Ma L, Zeng AP (2015) Determination of larval instars of the camellia weevil, Curculio chinensis (Coleoptera: Curculionidae). Acta Entomologica Sinica 58 (2): 181-189. https://doi.org/10.16380/j.kcxb.2015.02.011

- Lohse M, Drechsel O, Kahlau S, Bock R (2013) OrganellarGenomeDRAW — a suite of tools for generating physical maps of plastid and mitochondrial genomes and visualizing expression data sets. Nucleic Acids Research 41 (W1): W575-W581. https://doi.org/ 10.1093/nar/gkt289

- $\quad$ Meng GL, Li YY, Yang CT, Liu SL (2019) MitoZ: a toolkit for animal mitochondrial genome assembly, annotation and visualization. Nucleic Acids Research 47 (11): e63. https://doi.org/10.1093/nar/gkz173

- $\quad$ Miller MA, Pfeiffer W, Schwartz T (2010) Creating the CIPRES science gateway for inference of large phylogenetic trees. In proceedings of the gateway computing environments workshop (GCE). Institute of Electrical and Electronics Engineers (IEEE): New Orleans, LA, USA 14: 1-8.

- Minh BQ, Nguyen MAT, von Haeseler A (2013) Ultrafast approximation for phylogenetic bootstrap. Molecular Biology and Evolution 30 (5): 1188-1195. https://doi.org/10.1093/ molbev/mst024

- $\quad$ Narakusumo RP, Riedel A, Pons J (2020) Mitochondrial genomes of twelve species of hyperdiverse Trigonopterus weevils. PeerJ 8: e10017. https://doi.org/10.7717/peerj. 10017

- $\quad$ Nguyen LT, Schmidt HA, von Haeseler A, Minh BQ (2015) IQ-TREE: A fast and effective stochastic algorithm for estimating maximum-likelihood phylogenies. Molecular Biology and Evolution 32 (1): 268-274. https://doi.org/10.1093/molbev/msu300

- $\quad$ Ojala D, Montoya J, Attardi G (1981) tRNA punctuation model of RNA processing in human mitochondria. Nature 290: 470-474. https://doi.org/10.1038/290470a0

- Ronquist F, Teslenko M, van der Mark P, Ayres DL, Darling A, Hohna S, Larget B, Liu L, Suchard MA, Huelsenbeck JP (2012) MrBayes 3.2: efficient Bayesian phylogenetic inference and model choice across a large model space. Systematic Biology 61 (3): 539-542. https://doi.org/10.1093/sysbio/sys029

- $\quad$ Shu JP, Teng Y, Liu J, Zhang YB, Wang HJ (2013) Preliminary analysis on the causes of pre-harvest fruit drop in Camellia oleifera. China Plant Protection 33 (1): 9-14.

- $\quad$ Song N, Li XX, Yin XM, Li XH, Yin SJ, Yang MS (2020) The mitochondrial genome of Apion squamigerum (Coleoptera, Curculionoidea, Brentidae) and the phylogenetic implications. PeerJ 8: e8386. https://doi.org/10.7717/peerj.8386

- Talavera G, Castresana J (2007) Improvement of phylogenies after removing divergent and ambiguously aligned blocks from protein sequence alignments. Systematic Biology 56 (4): 564-577. https://doi.org/10.1080/10635150701472164

- $\quad$ van de Vossenberg BTLH, Warbroek T, Ingerson-Mahar J, Waalwijk C, van der Gouw LP, Eichinger B, Loomans AJM (2019) Tracking outbreak populations of the pepper weevil Anthonomus eugenii (Coleoptera; Curculionidae) using complete mitochondrial genomes. PLOS One 14: e0221182. https://doi.org/10.1371/journal.pone.0221182

- Wang BX, Xu YL, Zhuo ZH, Xu XL, Liu J, Qiu J, Fang R, Liu YK, Zeng Z, Xiao QG (2020) The complete mitochondrial genome of the fig weevil, Aclees cribratus 
(Coleoptera: Curculionidae). Mitochondrial DNA Part B 5 (3): 2599-2600. https://doi.org/ 10.1080/23802359.2020.1780978

- Wang BX, Zhuo ZH, Fang R, Yang H, Zhang DJ, Zhang BL, Sui LY, Ma WJ, Yang MF, Yang W (2021) Complete mitochondrial genome of the olive weevil, Dyscerus cribripennis (Coleoptera: Curculionidae). Mitochondrial DNA Part B 6 (1): 43-44. https://doi.org/10.1080/23802359.2020.1845580

- Wei SJ, Shi M, Chen XX, Sharkey MJ, van Achterberg C, Ye GY, He JH (2010) New views on strand asymmetry in insect mitochondrial genomes. PLOS One 5 (9): e12708. https://doi.org/10.1371/journal.pone.0012708

- Xu YD, Guan DL, Xu SQ (2017) Characterization of the complete mitochondrial genome of the chestnut weevil Curculio davidi (Insecta: Coleoptera: Curculionidae). Conservation Genetics Resources 9 (2): 285-288. https://doi.org/10.1007/s12686016-0674-0

- Z Zhang D, Gao FL, Jakovlić I, Zou H, Zhang J, Li WX, Wang GT (2019) PhyloSuite: an integrated and scalable desktop platform for streamlined molecular sequence data management and evolutionary phylogenetics studies. Molecular Ecology Resources 20 (1): 348-355. https://doi.org/10.1111/1755-0998.13096

- $\quad$ Zhou N, Dong YL, Qiao PP, Yang ZF (2020) Complete Mitogenomic Structure and Phylogenetic Implications of the Genus Ostrinia (Lepidoptera: Crambidae). Insects 11 (4): 232. https://doi.org/10.3390/insects11040232

\section{Supplementary materials}

\section{Suppl. material 1: Table S1 doi}

Authors: Kai Hu, Zaihua Yang

Data type: docx

Brief description: The best partitioning schemes and substitution models for PCG123 + rRNA dataset comprising 13 PCGs and two rRNAs of six species of Curculioninae used for ML phylogenetic analyses.

Download file $(17.00 \mathrm{~kb})$

\section{Suppl. material 2: Table S2 doi}

Authors: Kai Hu, Zaihua Yang

Data type: docx

Brief description: The best partitioning schemes and substitution models for PCG123 + rRNA dataset comprising 13 PCGs and two rRNAs of six species of Curculioninae used for $\mathrm{BI}$ phylogenetic analyses.

Download file $(15.73 \mathrm{~kb})$ 